\title{
Original
}

\section{Influence of Anatomical Variation in the Nasal Cavity on Inflammation of the Paranasal Sinuses}

\author{
Go TAKAhashi ${ }^{1)}$, Hajime Terao ${ }^{2)}$, Kiyoaki KamaKaZu ${ }^{3)}$, \\ Takeyuki SAnBE $^{3)}$, Isao SuZAKi ${ }^{1)}$, Toshimitsu KomatsuZAKI ${ }^{1)}$, \\ Ayako ITOH $^{1)}$ and Harumi SuZAKI ${ }^{1)}$
}

\begin{abstract}
We investigated the correlation between the incidence of anatomical variations in the nasal cavity and mucosal inflammation in the paranasal sinuses, using computed tomography (CT). In total, 239 patients (478 sides; 138 men and 101 women; age range, 8-89 years) underwent coronal plane CT for screening from November 2001 to October 2006. Patients with facial trauma, paranasal sinus carcinoma, inverted papilloma, or previous sinus surgery were excluded from this study. We evaluated the incidence of agger nasi air cells, Haller's cells, middle and superior turbinate pneumatization, paradoxically curved uncinate processes, paradoxically curved middle turbinates, and septal deviation. The mucosal condition and ostiomeatal complex were evaluated by the Lund-Mackay staging system, and correlations between groups were analyzed using Mann-Whitney's $U$ tests. The incidence of nasal septal deviation was $14.6 \%$ and the incidences of agger nasi air cells, concha bullosa (pneumatization of the middle nasal turbinate) and Haller's cells were $47.7 \%, 22.4 \%$ and $10.7 \%$, respectively. Paranasal CT showed partial or total opacification of the sinuses in approximately $40 \%$ of the anterior and posterior ethmoid and maxillary sinuses. Concha bullosa increased the CT opacification of the paranasal sinuses, except for the sphenoid sinus. There was no significant association between the occurrence of concha bullosa and nasal septal deviation. Our results suggest anatomical variations in the nasal cavity induce mucosal inflammation in the paranasal sinuses.
\end{abstract}

Key words : anatomical variations, computerized tomography of the paranasal sinuses, chronic sinusitis, ostiomeatal complex

\section{Introduction}

Chronic sinusitis is defined as prolonged inflammation in the paranasal sinus for 3 months or longer. Prolongation of inflammation in the paranasal sinus is associated with anatomical

\footnotetext{
1) Department of Otorhinolaryngology, Showa University School of Medicine, 1-5-8 Hatanodai, Shinagawa-ku, Tokyo 142-8666, Japan.

2) Department of Otorhinolaryngology, Ebara Hospital, Tokyo Metropolitan Health and Medical Treatment Corporation.

3) Department of Otorhinolaryngology, Showa University Fujigaoka Hospital.
} 
abnormalities such as nasal septal deviation, inflammation of the mucosa of the nasal cavity caused by complications from nasal allergy and bronchial asthma, and infection by viruses, bacteria and fungi. In particular, the ostiomeatal complex (OMC) provides a ventilation route from the paranasal sinus (mainly the anterior group of paranasal sinuses), and obstructive disease in the $\mathrm{OMC}$ is an important factor in prolonging inflammation in the paranasal sinus. The major cause of OMC obstruction is nasal septal deviation, followed by anatomical variations in the lateral nasal wall around the anterior ethmoid sinus. Anatomical variations in the nasal and paranasal sinuses are often seen in daily practice, with an incidence of $93 \%$ found by Earwaker ${ }^{1)}$. However, the effect of anatomical variation in the nasal cavity on inflammation in the paranasal sinus remains unclear, and this effect was investigated in the current study.

\section{Materials and Methods}

This study included 239 patients with nasal symptoms who underwent coronal plane computerized tomography (CT) of the paranasal sinuses $(n=478)$ at Showa University Fujigaoka Hospital from November 2001 to October 2006. The patients comprised 138 males and 101 females aged 8 to 89 years old (mean, 51.2 years). Patients with previous sinus surgery, facial trauma, paranasal sinus tumors or congenital anomalies were excluded from the study. CT scans were performed in helical acquisition mode with tube voltage $120 \mathrm{kVp}$ and an autotube current setting at Noise Index 8.0 using 4- and 32-slice multislice CT (LightSpeed Plus and LightSpeed VCT-Select, GE Healthcare, WI). The images were reconstructed using the Bone reconstruction kernel with a $1.25-\mathrm{mm}$ image slice thickness and a $0.625-\mathrm{mm}$ image interval. Multiplanar reformatted images of the coronal plane of 5.0-mm thickness were subsequently made on a workstation (Advantage Workstation 4.3, GE Healthcare).

The anatomical variations examined in the study included agger nasi air cells, Haller's cells, concha bullosa, pneumatization of the superior nasal turbinate, paradoxical curve of the uncinate process, and paradoxical curve of the middle nasal turbinate. Nasal septal deviation, which was defined as a deviation in the OMC that influenced the surrounding structure, regardless of the position of the top of the deviation, was also investigated. Cases with a deviation in the inferior nasal septum but not in the OMC (e.g., a deviation forming a crista or spina at the bone-cartilage joint) were excluded. The presence of anatomical variations and nasal septal deviation was examined in coronal plane CT images of the paranasal sinuses. The severity of CT opacification in each part of the paranasal sinus and OMC was calculated and scored similar to CT image scoring, using the Lund-Mackay System $^{2)}$. The Lund-Mackay System is a standard CT scoring system for assessment of opacification of each sinus system and the OMC. CT opacification in the frontal sinus, anterior and posterior ethmoid sinuses, maxillary sinus and sphenoid sinus was scored as 0,1 or 2 points for normal, partial or total opacification, respectively. For the OMC, the absence or presence of obstruction was scored as 0 or 2 points, respectively. The relationships between 


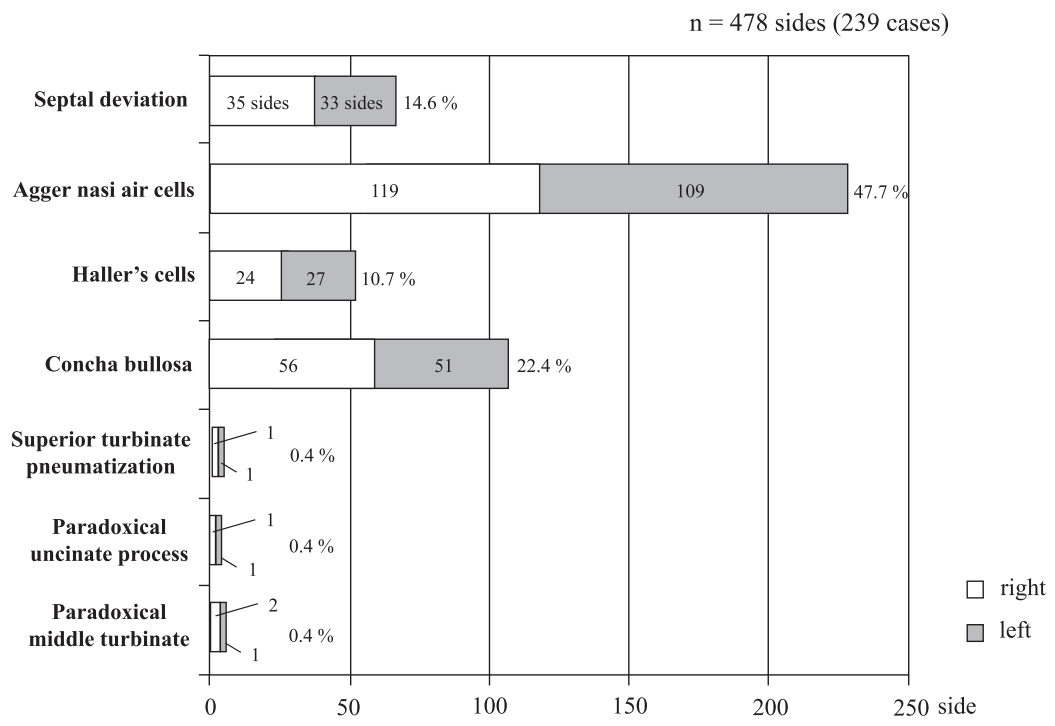

Fig. 1. Frequency of anatomical variations found using computerized tomography imaging in 239 patients with nasal symptoms.

CT opacification in the paranasal sinuses and OMC, and nasal septal deviation and anatomical variations were analyzed. The relationship between nasal septal deviation and concha bullosa was also evaluated. Statistical analyses were performed by the Mann-Whitney $U$ test and Fisher exact test, with the level of significance set at $P<0.05$.

\section{Results}

\section{Incidences of anatomical variations}

The incidences of nasal septal deviation and anatomical variations in all 239 patients (478 sides) are shown in Figure 1. These incidences were as follows: nasal septal deviation, $14.6 \%$ (35 on the right side, 33 on the left side, 68 in total; Fig. 2); agger nasi air cells, 47.7\% (119 right, 109 left, 228 total ; Fig. 3); Haller's cells, 10.7\% (24 right, 27 left, 51 total ; Fig. 4); concha bullosa, 22.4\% (56 right, 51 left, 107 total ; Fig. 5); pneumatization of the superior nasal turbinate, $0.4 \%$ (1 right, 1 left, 2 total; Fig. 6); paradoxical curve of the uncinate process, $0.4 \%$ (1 right, 1 left, 2 total; Fig. 7); and paradoxical curve of the middle nasal turbinate, $0.6 \%$ (2 right, 1 left, 3 total ; Fig. 8).

\section{Severity of inflammation in the paranasal sinuses and OMC}

Severity scores for CT opacification in the paranasal sinuses and OMC in 239 patients (478 sides) are shown in Figures 9 and 10. In the frontal sinus, scores of 0,1 and 2 points were found in 368, 30 and 80 sides, respectively (right frontal sinus: 185, 14, and 40; left frontal sinus: 183, 16, and 40, respectively); and in the anterior ethmoid sinus, 257, 111 and 110 sides scored 0, 1, and 2 points, respectively, (right: 127, 59, and 53; left : 130, 52, and 57, 


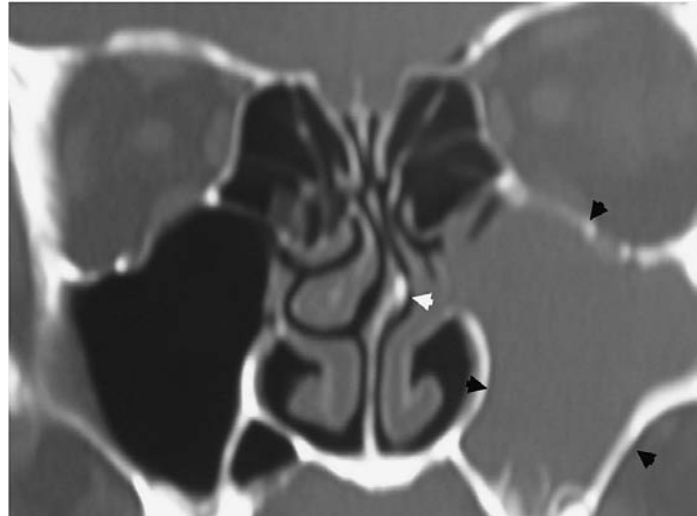

Fig. 2. Computerized tomography findings in a patient with nasal septal deviation (white arrows) that caused occlusion of the ostiomeatal complex and produced inflammation in the left maxillary sinus (black arrows).

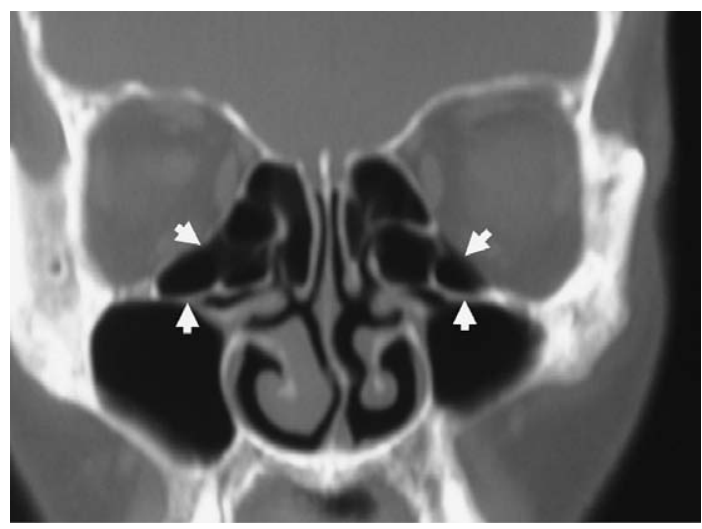

Fig. 4. Computerized tomography findings in a patient with bilateral Haller' s cells (white arrows).

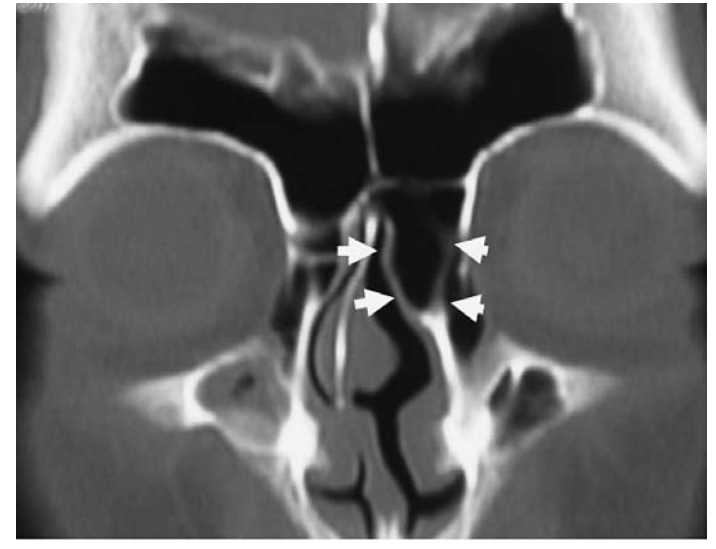

Fig. 3. Computerized tomography findings in a patient with large agger nasi air cells (white arrows) observed as a prominence in the left lateral nasal wall.

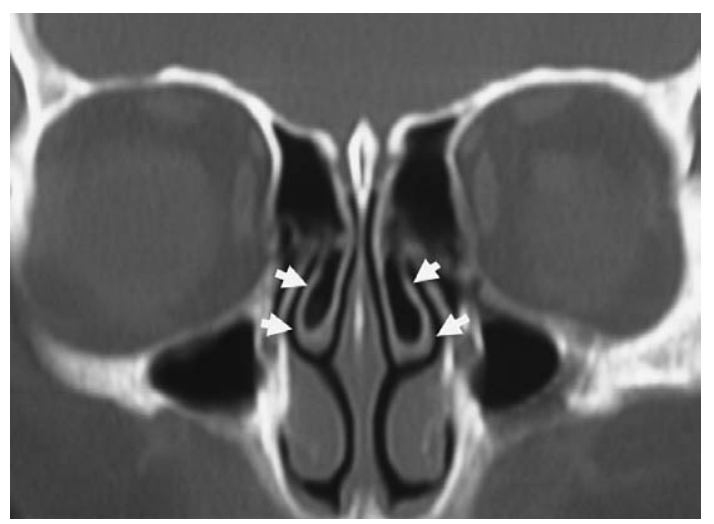

Fig. 5. Computerized tomography findings in a patient with bilateral middle nasal turbinate pneumatization (concha bullosa) (white arrows).

respectively). Scores of 0,1 and 2 points were found in 286, 135 and 57 sides, respectively, of the posterior ethmoid sinus (right: 141, 69, and 29; left : 145, 66, and 28, respectively); and 400, 48 and 30 sides, of the sphenoid sinus (right: 197, 26, and 16; left: 203, 22, and 14); while in the maxillary sinus, 270, 142 and 66 sides scored 0,1 and 2 points (right: 134, 75, and 30; left: 136, 67, and 36). In the OMC, scores of 0 and 2 points were given for 270 and 208 sides (right: 135, and 104; left: 135, and 104).

\section{Association of anatomical variations with inflammation in the paranasal sinuses and OMC}

The influence of anatomical variation in the nasal cavity on inflammation of the paranasal sinuses was evaluated by analysis of the correlation between the incidence of the variations 


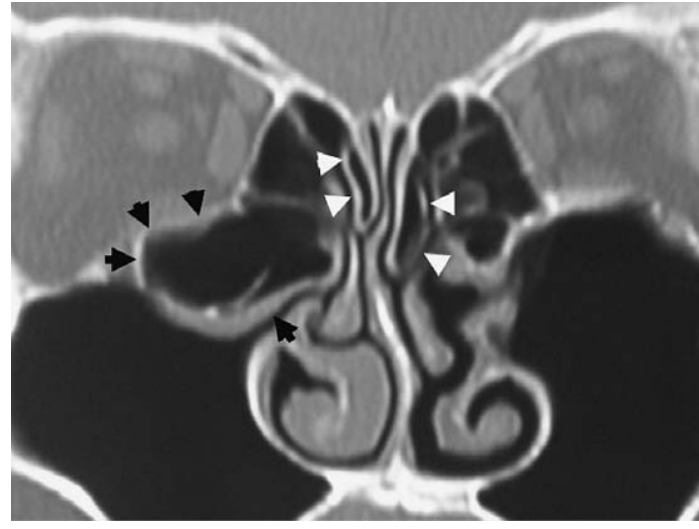

Fig. 6. Computerized tomography findings in a patient with bilateral superior nasal turbinate pneumatization (white arrowheads). Haller's cells (black arrows) on the right side, nasal septal deviation and a hypertrophied inferior nasal turbinate are also seen.

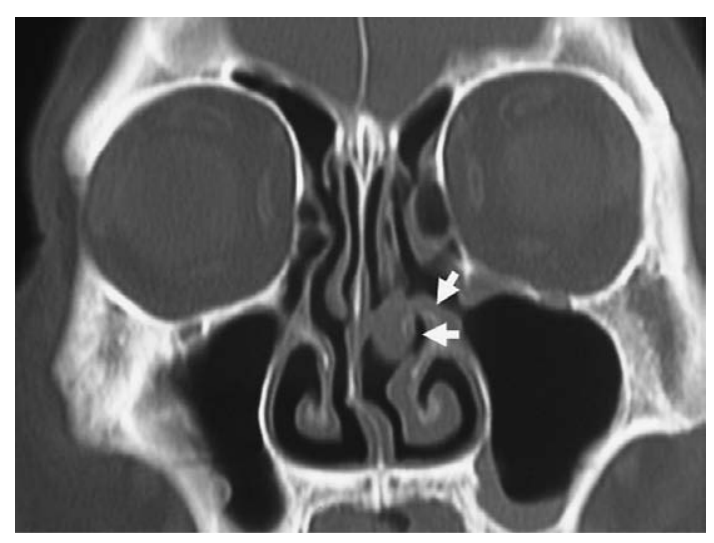

Fig. 7. Computerized tomography findings in a patient with a paradoxical (curved) uncinate process (white arrows). The left uncinate process protrudes toward the lower inner part of the nasal cavity.

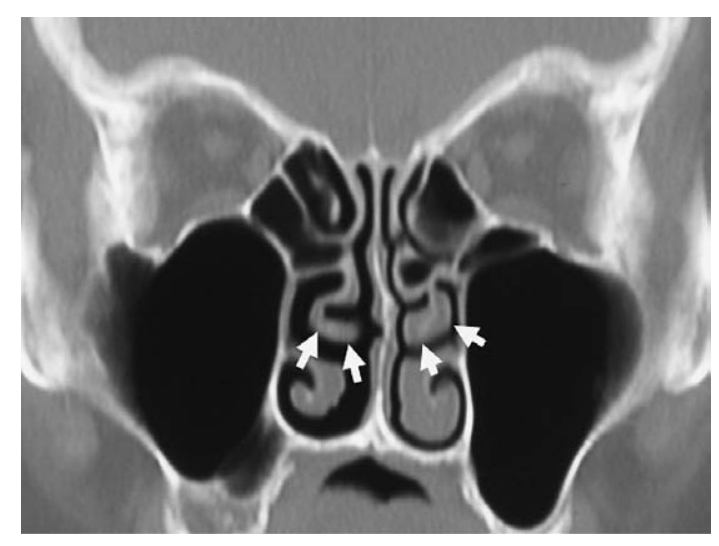

Fig. 8 Computerized tomography findings in a patient with a paradoxical (curved) middle nasal turbinate (white arrows). The tips of the bilateral middle nasal turbinates curve toward the nasal septum.

and the scores for CT opacification in the paranasal sinuses and OMC. Correlations of the incidences of nasal septal deviation, agger nasi air cells, concha bullosa, and Haller's cells with the CT opacification scores for the frontal sinus, anterior and posterior ethmoid sinuses, maxillary sinus, sphenoid sinus and $\mathrm{OMC}$ are shown in Table 1. Statistical analyses were performed by Mann-Whitney $U$ tests. Significant correlations were found between nasal septal deviation and CT opacification in the anterior ethmoid sinus and the OMC (both $P$ $<0.05)$; and between agger nasi air cells and CT opacification in the anterior and posterior ethmoid sinuses (both $P<0.01)$ and the OMC $(P<0.05)$. Middle turbinate pneumatiza- 


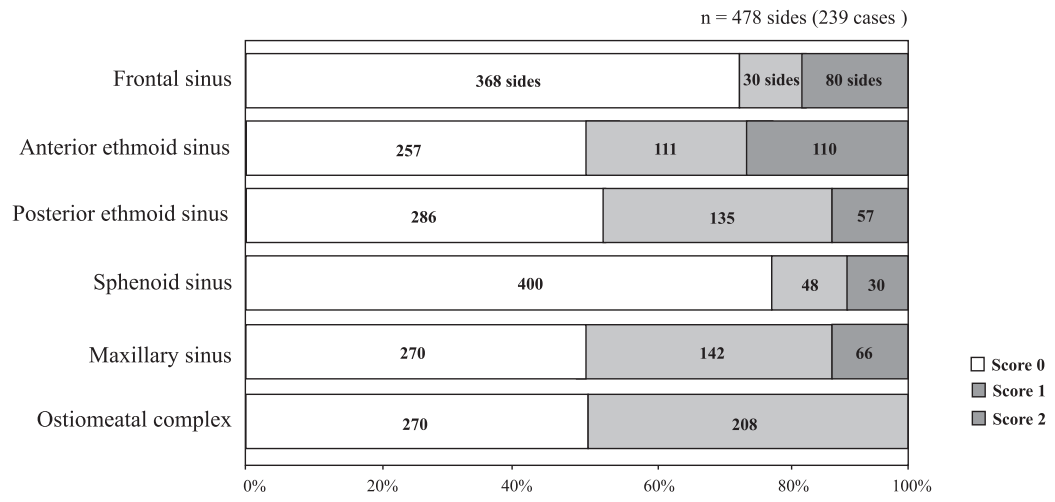

Fig. 9 Computerized tomography opacification scores for the paranasal sinus (left and right sides combined) and ostiomeatal complex.
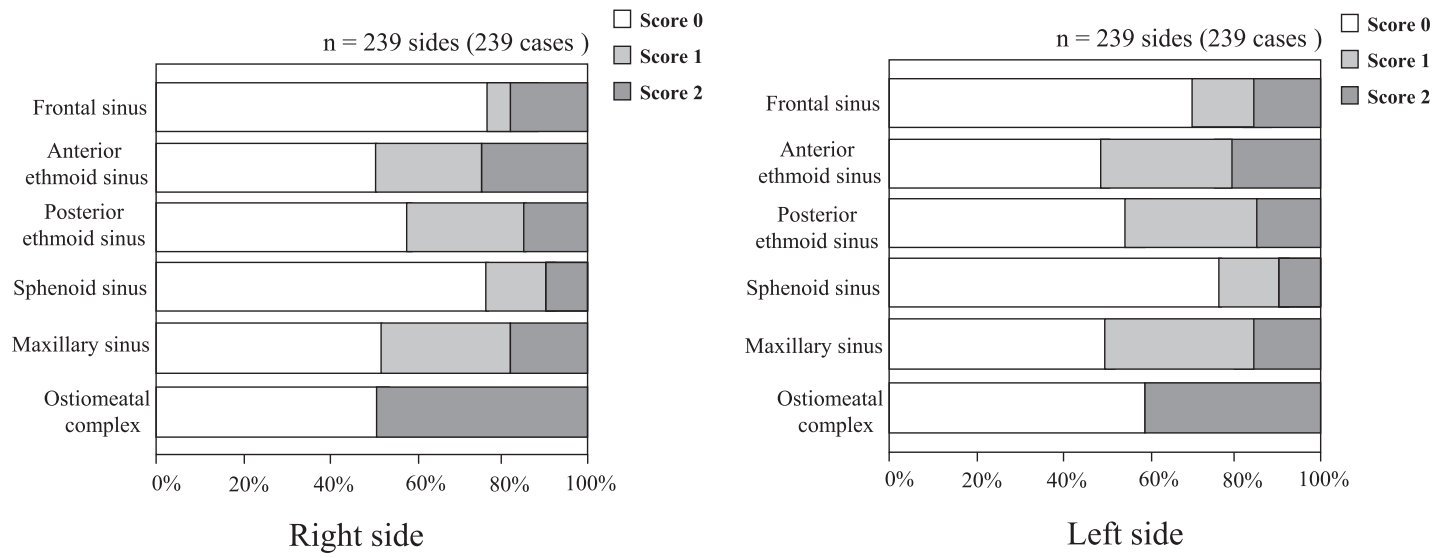

Fig. 10 Computerized tomography opacification scores for each side of the paranasal sinus and ostiomeatal complex.

tion was also significantly correlated with $\mathrm{CT}$ opacification in the frontal, anterior and posterior ethmoid sinuses and the OMC (all $P<0.01$ ) and the maxillary sinus $(P<0.05)$. In addition, the presence of Haller's cells was significantly correlated with CT opacification in the frontal, anterior and posterior ethmoid sinuses (all $P<0.05$ ).

\section{Association between concha bullosa and nasal septal deviation}

We compared the frequency of occurrence of concha bullosa with nasal septal deviation with concave or convex surfaces. The Fisher exact test gave a $P$-value of 0.28 , indicating no significant association between the occurrence of concha bullosa and nasal septal deviation.

\section{Discussion}

The primary goal of endoscopic endonasal sinus surgery for chronic sinusitis is to remove obstructive lesions in the $\mathrm{OMC}$ and improve the function of mucociliary transportation in 
Table 1. The relationships between anatomical variations and computerized tomography opacification scores for the paranasal sinuses and ostiomeatal complex (OMC).

\begin{tabular}{ccccccc}
\hline & $\begin{array}{c}\text { Frontal } \\
\text { sinus }\end{array}$ & $\begin{array}{c}\text { Anterior } \\
\text { ethmoid } \\
\text { sinus }\end{array}$ & $\begin{array}{c}\text { Posterior } \\
\text { ethmoid } \\
\text { sinus }\end{array}$ & $\begin{array}{c}\text { Sphenoid } \\
\text { sinus }\end{array}$ & $\begin{array}{c}\text { Maxillary } \\
\text { sinus }\end{array}$ & OMC \\
\hline Septal deviation & NS & $*$ & NS & NS & NS & NS \\
Agger nasi air cells & NS & $* *$ & $* *$ & NS & $*$ & $*$ \\
$\begin{array}{c}\text { Middle turbinate } \\
\text { pneumatization }\end{array}$ & $* *$ & $* *$ & $* *$ & NS & NS & NS \\
Haller's cells & $*$ & $*$ & $*$ & & & $*$ \\
\hline
\end{tabular}

**, $P<0.01 ;{ }^{*}, \mathrm{P}<0.05 ; \mathrm{NS}$, not significant.

the paranasal sinus. The $\mathrm{OMC}$ is not an anatomical region, but rather represents the opening site in the nasal cavity including the middle meatus, bulla ethmoidalis, uncinate process, ethmoidal infundibulum, semilunar hiatus, and the anterior group of paranasal sinuses; i.e., the frontal, anterior ethmoid and maxillary sinuses. These regions are narrow and small, and therefore tend to be obstructed by mucosal swelling and polyps. The OMC is a clinically important route for ventilation and excretion in the paranasal sinus and is associated with development and prolongation of sinusitis. Therefore, the properties of the OMC are important in endoscopic endonasal sinus surgery for opening of this region.

In the 1970's, a hypothesis was proposed that inflammation in the paranasal sinus was caused by anatomical and functional abnormalities in the lateral nasal wall ${ }^{3)}$. This hypothesis suggested that inflammation in the paranasal sinus developed in the nasal cavity and caused swelling in the middle meatus mucosa and dysfunction in mucociliary transportation, resulting in sinusitis. At that time, inflammation in the anterior ethmoid sinus was thought to cause obstruction in the ethmoidal infundibulum and frontal recess, with consequent hypoventilation of the maxillary and frontal sinuses. Inflammation in the maxillary and frontal sinuses was thought to cause hypofunction of mucociliary transportation, resulting in chronic inflammatory disease. However, it is unclear how anatomical variation affects inflammation in the paranasal sinus. Stammberger ${ }^{3)}$ proposed that anatomical variations were important in chronic sinusitis, whereas Lloyd et $a l^{4,5)}$ and Jones et $a l^{6)}$ concluded that these variations had no effect on swelling in the paranasal sinus mucosa based on the lack of correlation between such swelling and the incidence of anatomical variations.

Arslan $e t ~ a l^{7)}$ reported a $36 \%$ incidence of nasal septal deviation in patients with sinusitis while Earwaker ${ }^{1)}$ found an incidence of $79 \%$. Incidences of $20 \%$ and $24 \%$ have been reported for nasal septal deviation in the OMC alone ${ }^{6,8)}$. The substantial difference in incidence rates is due to the different definitions used for nasal septal deviation among these studies: a higher incidence is obtained when the definition of nasal septal deviation includes the entire region. In the current study, our definition of septal deviation required that the 
top of the deviation was in the OMC. This led to an incidence of deviation of $14 \%$, with an association with inflammation in the anterior ethmoid sinus and OMC. Several studies investigating the association between nasal septal deviation and sinusitis have indicated that more severe nasal septal deviation is related to an increased incidence and severity of sinusitis $^{8-12)}$. However, these studies did not indicate whether sinusitis developed on the same or opposite side to that of the nasal septal deviation. Frenkiel and Fageeh ${ }^{13)}$ showed that the incidence of obstruction in the OMC was higher on the concave side of the nasal septal deviation, and that anatomical variations of the lateral nasal wall and the middle turbinate were induced on the concave side.

Concha bullosa is caused by pneumatization in the middle and upper turbinates and swelling, with most cases developing in the middle turbinate. We identified concha bullosa in $22 \%$ of patients, with an association with mucosal thickening in the frontal, anterior ethmoid and maxillary sinuses and the OMC. Arslan and colleagues ${ }^{7)}$ reported a $30 \%$ incidence of concha bullosa in patients with sinusitis, while two other studies in patients with or without sinusitis reported rates of $28 \%$ to $34 \%$ and concluded that there was no difference in the incidence of OMC obstruction and severity of sinusitis regardless of the presence of concha bullosa ${ }^{14,15)}$. Scribano and Colleagues ${ }^{16)}$ found concha bullosa in 67 of 146 sides $(46 \%)$ and a maxillary lesion on the same side was found in 17 of these cases. Another study ${ }^{8)}$ reported that concha bullosa in patients with sinusitis $(n=100)$ showed a significantly higher incidence and stronger correlation with mucosal thickening in the anterior ethmoidal sinus, compared to patients without sinusitis $(n=82)$, based on a comparison of CT images.

In an investigation by Tonai and $\mathrm{Baba}^{15)}$, agger nasi air cells were found in $86 \%$ of patients with sinusitis and in $89 \%$ of those without sinusitis, while other studies have found that almost all patients with sinusitis have agger nasi air cells ${ }^{17,18)}$. Our results showed an incidence of agger nasi air cells of $48 \%$, with an association with inflammation in the anterior and posterior ethmoid sinuses and the OMC. Haller's cells are ethmoidal cells that develop posterosuperiorly in the maxillary sinus and extend from the maxillary sinus to the orbital floor. Haller's cells have potential effects on the OMC due to the site of development. Scribano et $a l^{16)}$ found Haller's cells in 24 of 146 sides (16\%) in patients with or without sinusitis, while other studies have found the incidence of Haller's cells to range from $6 \%$ to $33 \%$ in patients with sinusitis, and $6 \%$ to $39 \%$ in those without sinusitis, with the incidence showing no dependence on the presence or severity of sinusitis. Our results showed an $11 \%$ incidence of Haller's cells, with an association with inflammation in the frontal, anterior and posterior ethmoid sinuses and the OMC.

Pneumatization of the superior nasal turbinate is an anatomical variation included in concha bullosa. Earwaker described this variation in 98 of 800 patients, with 34 cases being bilateral $^{1 \text { ) }}$, but we only found an incidence of $0.4 \%$. The incidence of paradoxical (curved) uncinate process was also $0.4 \%$ in our patients, and has not been reported in previous stud- 
ies. For paradoxical (curved) middle nasal turbinate, we found an incidence of $0.6 \%$. Two previous studies found no difference in the incidence of this variation in the absence or presence of sinusitis ${ }^{8,17)}$. Overall, of the anatomical variations examined in the current study, nasal septal deviation, concha bullosa and agger nasi air cells had a significant association with OMC obstruction, which suggests that these variations prolong inflammation in the paranasal sinus. Smokers and patients with conditions such as allergic rhinitis and bronchial asthma have chronic inflammation in the nasal mucosa that may exacerbate the effects of anatomical variations.

\section{Conclusion}

Our results suggest that anatomical variations in the nasal cavity are associated with the disease status of sinusitis. These anatomical variations are associated with obstruction of narrow and complex structures in the OMC and are likely to cause stenosis and obstruction in the natural ostium of the paranasal sinus. This may lead to disturbance of ventilation and excretion, resulting in prolongation of inflammation in the paranasal sinus.

\section{References}

1) Earwaker J : Anatomic variants in sinonasal CT. Radiographics 13 : 381-415 (1993)

2) Lund VJ and Mackay IS : Staging in rhinosinusitis. Rhinology 31:183-184 (1993)

3) Stammberger H: Endoscopic endonasal surgery-concepts in treatment of recurring rhinosinusitis. Part I. Anatomic and pathophysiologic considerations. Otolaryngol Head Neck Surg 94 : 143-147 (1986)

4) Lloyd GA : CT of the paranasal sinuses: study of a control series in relation to endoscopic sinus surgery. $J$ Laryngol Otol $104: 477-481$ (1990)

5) Lloyd GAS, Lund VJ and Scadding GK: CT of the paranasal sinuses and functional endoscopic surgery: a critical analysis of 100 symptomatic patients. J Laryngol Otol 105 : 181-185 (1991)

6) Jones NS, Strobl A and Holland I: A study of the CT findings in 100 patients with rhinosinusitis and 100 controls. Clin Otolaryngol 22 : 47-51 (1997)

7) Arslan H, Aydinlioglu A, Bozkurt M and Egeli E: Anatomic variations of the paranasal sinuses : CT examination for endoscopic sinus surgery. Auris Nasus Larynx 26:39-48 (1999)

8) Calhoun KH, Waggenspack GA, Simpson CB, Hokanson JA and Bailey BJ : CT evaluation of the paranasal sinuses in symptomatic and asymptomatic populations. Otolaryngol Head Neck Surg 104: 480-483 (1991)

9) Danese M, Duvoisin B, Agrifolio A, Cherpillod J and Krayenbulh M: Influence des variantes anatomicques nasosinusales sur res sinusites recidivantes, persistantes ou chroniques, Evaluation TDM chez 112 patients. $J$ Radiol $78:$ 651-657 (1997)

10) Yousem DM, Kennedy DW and Rosenberg S: Ostiomeatal complex risk factors for sinusitis : CT evaluation. $J$ Otolaryngol 20 : 419-424 (1991)

11) Matschke RG and Fiebach A : Septumdeviation und begleitsinusitis. HNO 133 : 541-544 (1985)

12) Elahi MM and Frenkiel S : Septal deviation and chronic sinus disease. Am J Rhinol 14: 175-179 (2000)

13) Elahi MM, Frenkiel $\mathrm{S}$ and Fageeh $\mathrm{N}$ : Paraseptal structural changes and chronic sinus disease in relation to the deviated septum. J Otolaryngol $26: 236-240$ (1997)

14) Zinreich SJ, Mattox DE, Kennedy DW, Chisholm HL, Diffley DM and Rosenbaum AE: Concha bullosa : CT evaluation. J Comput Assist Tomogr 12 : 778-784 (1988)

15) Tonai A and Baba S: Anatomic variations of the bone in sinonasal CT. Acta Otolaryngol 525(Suppl): 9-13 (1996) 
16) Scribano E, Ascenti G, Loria G, Cascio F and Gaeta $M$ : The role of the ostiomeatal unit anatomic variations in inflammatory disease of the maxillary sinuses. Eur J Radiol $24: 172-174$ (1997)

17) Bolger WE, Butzin CA and Parsons DS : Paranasal sinus bony anatomic variations and mucosal abnormalities: CT analysis for endoscopic sinus surgery. Laryngoscope 101 : 56-64 (1991)

18) Kennedy DW and Zinreich SJ: The functional endoscopic approach to inflammatory sinus disease : current perspective and technique modifications. Am J Rhinol 2:89-96 (1988)

[Received April 19, 2012 : Accepted May 16, 2012] 